\title{
MONOLITHIC PREAMPLIFIER EMPLOYING EPITAXIAL N-CHANNEL JFETS
}

\author{
BNL- -48005 \\ V. Radeka, S. Rescia \\ DE93 002951 \\ Brookhaven National Laboratory, Upton, NY 11973 \\ P.F. Manfredi, V. Re, V. Speziali \\ Dipartimento di Elettronica, Università di Pavia, Via Abbiategrasso 209, 27100 \\ Pavia Italy, and INFN, Sezione di Milano, Via Celoria 16, 20133 Milano, Italy
}

\begin{abstract}
This paper reports the results obtained in the research program oriented to the realisation of a monolithic preamplifier for calorimetry applications at high luminosity colliders.

The main purpose of the program is to arrive at a monolithic realisation with a performance as close as possible to that of discrete preamplifiers.

The junction field-effect transistors employed in discrete preamplifiers have an epitaxial channel and a very heavily doped gate diffused onto it. They present the best noise and radiation tolerance characteristics.

The first step in the program implementation was, accordingly, the search for a process able to make the integration of epitaxial-channel JFETs on a monolithic substrate possible.

The integration has been accomplished on the basis of a buried-layer approach to device isolation. Individual JFETs and a complete preamplifier employing only $\mathrm{N}$ channel JFETs have been realised.

The characterisation of the individual devices has shown that their behaviour in terms of small signal and noise parameters is very close to that of their discrete equivalents. This result, along with the very good noise performances of the preamplifier, seems to point out that the buried layer process has fulfilled the task for which it was developed.
\end{abstract}




\section{I-INTRODUCTION}

Low-noise signal amplification in calorimetry applications at high luminosity colliders requires front-end devices that, besides low noise performances, have also an adequate degree of radiation hardness.

The discrete N-channel junction field-effect transistors (JFET) ordinarily realised by diffusing a highly doped P-type region, which constitutes the gate, into an epitaxial $\mathrm{N}$-doped channel meets both requirements[1,2]. JFETs obtained with such a process will be referred to in what follows as epitarial-channel JFETs.

It can be understood, therefore, why in a program oriented to a monolithic preamplifier for calorimetry applications, with the goal of approaching to the best possible extent the noise and radiation hardness characteristics of a discrete design, the JFETs based on the previously outlined process were considered the most suitable devices.

As a preliminary step, a considerable effort had to be produced in the search for a technological process able to integrate epitaxial-channel JFETs into a monolithic structure without degrading their noise performance. This problem was eventually solved by using a buried layer technique to define the isolation tubs in which the JFETs are realised [3].

JFET parts obtained on a buried-layer isolated monolithic structures were proven to be very close to their discrete equivalents in both respects, small-signal behaviour and noise characteristics.

A monolithic preamplifier structure was then realised on the base of the buriedlayer process. Its configuration is given in fig. 1a), while fig. 1b) shows the off-thechip passive components that complete the charge-sensitive configuration. In the present version, auxiliary bonding pads have been added in order to connect some internal circuit nodes, of particular interest in the preliminary tests, to external pins on the package.

In this way the behaviour of the individual components as well as the complete monolithic preamplifier has been thoroughly characterised and the results of the measurements will be discussed in this paper. 


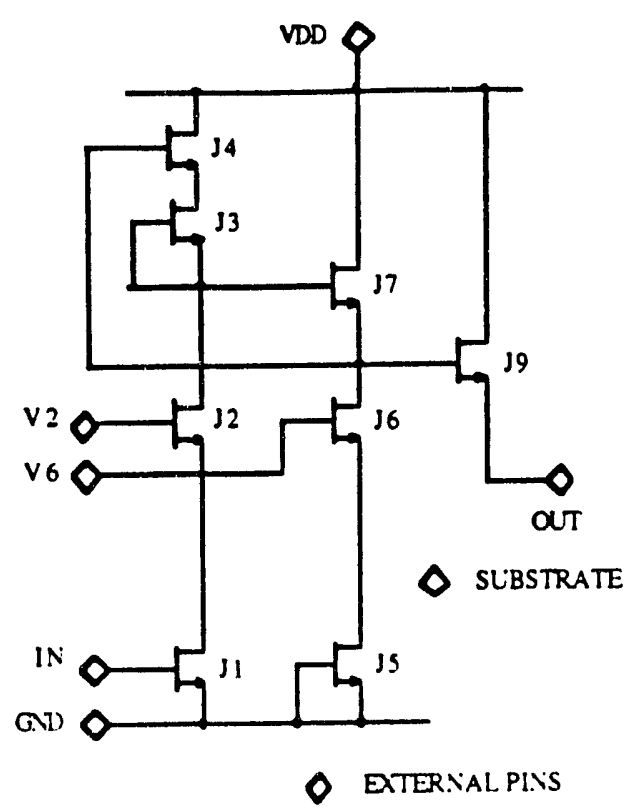

a)

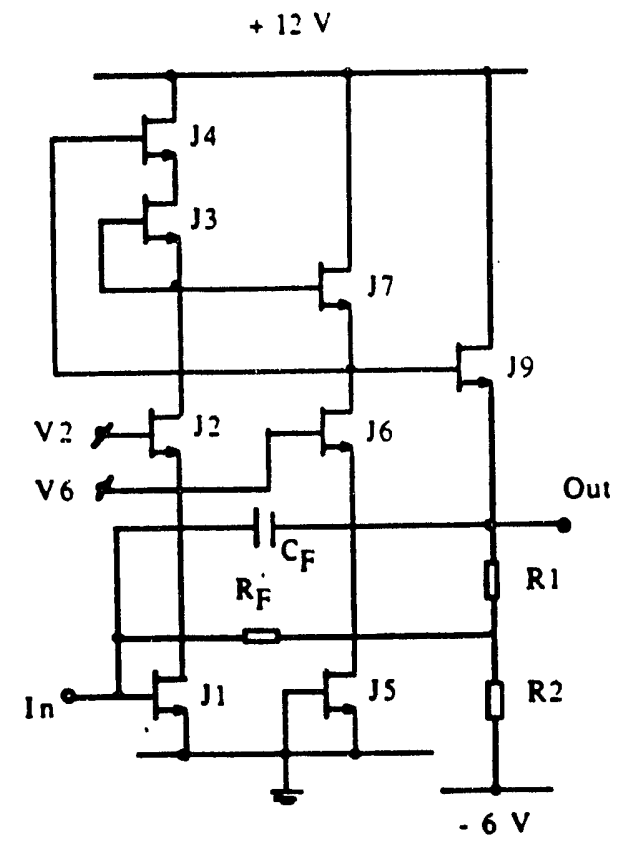

b)

Fig. 1- a) Monolithic preamplifier structure.

b) Charge-sensitive configuration showing the externally added components. 


\section{II- PREAMPLIFIER CHARACTERISTICS}

The basic features of the monolithic preamplifier structure are summarised in Table I.

The power dissipation largely exceeds the design value, as a consequence of the fact that in the considered technological run the value of the process pinch-off voltages was higher than specified. This results in a lower $\mathrm{g}_{\mathrm{m}} / \mathrm{I}$ DSS ratio and in higher reference $\mathrm{I}_{\mathrm{DSS}}$ currents, exceeding the target values of more than a factor two. Lower pinch-off voltages should bring down the power dissipation to less than $100 \mathrm{~mW}$.

\section{III - SMALL SIGNAL BEHAVIOUR}

The open-loop gain and phase characteristics are plotted as functions of frequency in fig. 2a), while fig. 2b) shows the effect of the substrate voltage on the gain characteristics.

a)

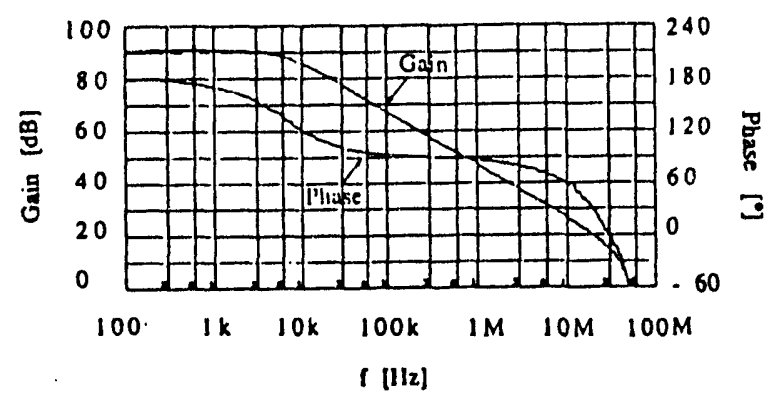

b)

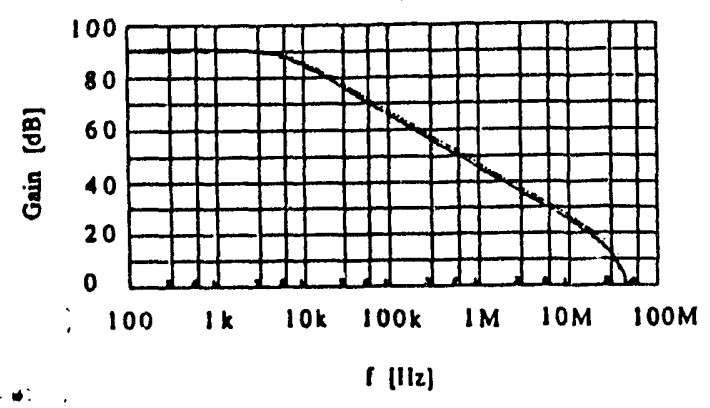

Fig. 2 - a) Open-loop gain and phase characteristics as functions of frequency.

b) Open-loop gain characteristics at two different values of the substrate voltage (continuous line: $\mathrm{V}_{\text {substrate }}=+12 \mathrm{~V}$; dotted line: $\mathrm{V}_{\text {substrate }}=+20 \mathrm{~V}$ ). 
The plots of fig. 2 show that both gain and phase characteristics have a very regular behaviour and that the preamplifier has a dominant pole at a frequency of about $6 \mathrm{kHz}$ and a second pole at about $15 \mathrm{MHz}$.

The response to a delta-impulse input current is shown in fig. 3 for a $C_{D}$ value of $500 \mathrm{pF}$. The $10 \%$ to $90 \%$ risetime is about $37 \mathrm{~ns}$. As for the gain-bandwidth product, an improvement in the risetime is expected in the second version of the preamplifier after the test pads and relevant connections to external pins, that add considerable parasitic capacitance, are removed.

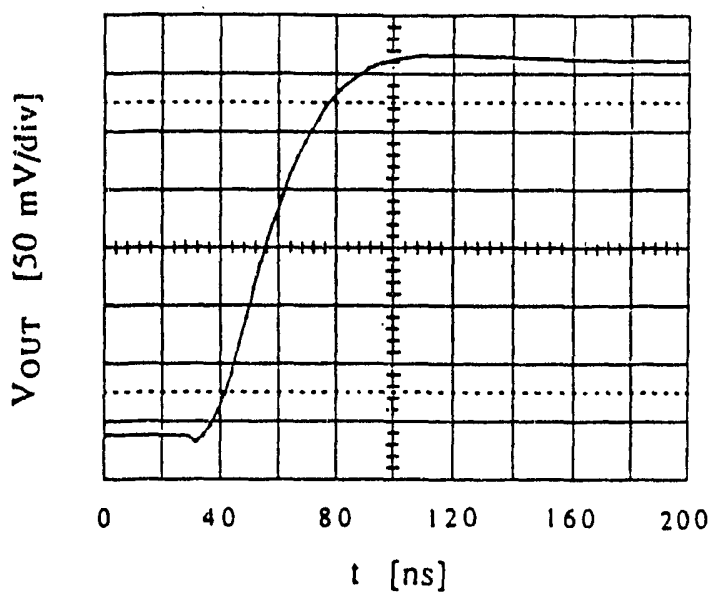

Fig. 3 - Preamplifier response to a delta-impulse input current for a $500 \mathrm{pF}$ detector capacitance and a $10 \mathrm{pF}$ feedback capacitance (for $\mathrm{C}_{\mathrm{F}}=33 \mathrm{pF}$ used in the tests of fast liquid argon calorimeters, the time scale is reduced by a factor of 3.3).

\section{IV - NOISE CHARACTERISATION}

The noise voltage spectral density referred to the preamplifier input is plotted as a function of frequency in fig. 4. 


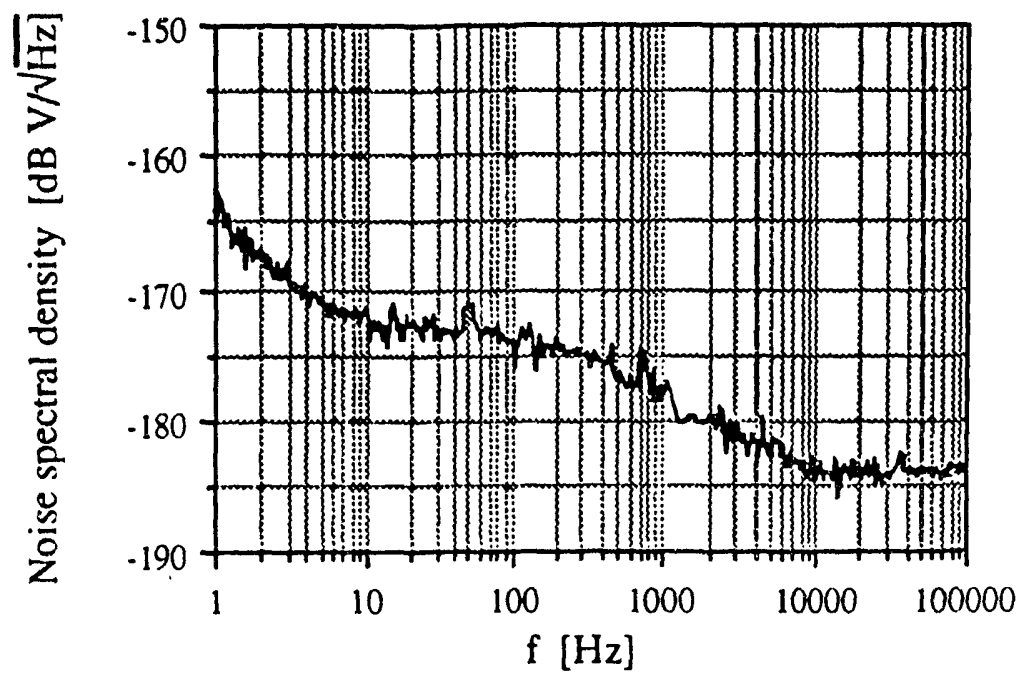

Fig. 4 - Spectral density of the noise voltage referred to the preamplifier input as a function of frequency $(0 \mathrm{~dB}$ corresponds to $1 \mathrm{~V} / \sqrt{\mathrm{Hz}})$. At f $>10^{4} \mathrm{~Hz} \mathrm{e}_{\mathrm{n}} \cong 0.63 \mathrm{nV} / \sqrt{\mathrm{Hz}}$.

As shown in fig. 4 , the voltage density in the white noise region is $-184 \mathrm{~dB}$, i.e. $e_{n} \equiv 0.63 \mathrm{nV} / \sqrt{\mathrm{Hz}}$. The theoretical noise voltage density corresponding to the thermal noise in the channel of the input device alone (measured $\mathrm{g}_{\mathrm{m}}=48.5 \mathrm{~mA} / \mathrm{V}$ ) would be $-186.37 \mathrm{~dB}$. This means that the excess noise in the first transistor and the white noise contribution coming devices other than the input one is $2.37 \mathrm{~dB}$.

The behaviour of the preamplifier in terms of the equivalent noise charge is described by the two plots in fig. 5 .

Section a) in fig. 5 shows the dependence of ENC on the peaking time $t_{p}$ of a semigaussian shaping amplifier with the detector capacitance $C_{D}$ as a parameter. At a $480 \mathrm{pF}-\mathrm{C}_{\mathrm{D}}$, values of ENC around $3700 \mathrm{rms}$ electrons are obtained with a peaking time of $200 \mathrm{~ns}$. This would result in $11700 \mathrm{rms}$ electrons at $t_{p}=20 \mathrm{~ns}$.

According to fig. $5 \mathrm{~b}$ ), a value of the $\mathrm{dENC} / \mathrm{dC}_{\mathrm{D}}$ sensitivity of $2.8 \mathrm{e} / \mathrm{pF}$ is obtained at a peaking time of $1 \mu \mathrm{s}$. This is very close to the slope which is theoretically expected under the hypothesis that the only noise term in the preamplifier is the thermal noise in the channel of the input device. 
a)

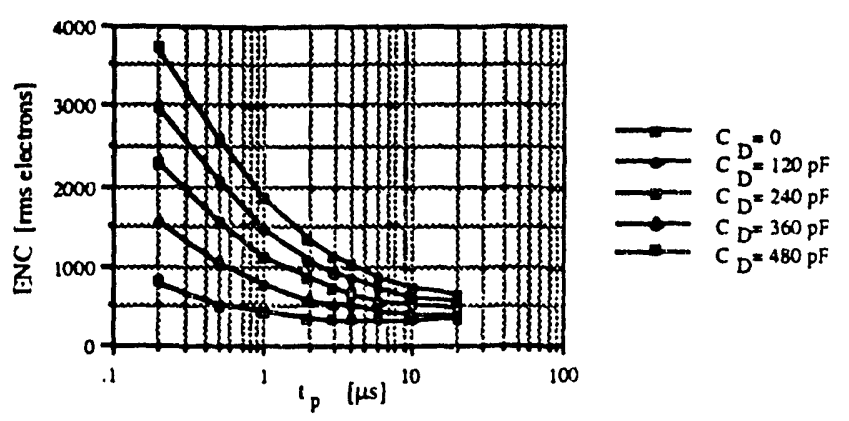

b)

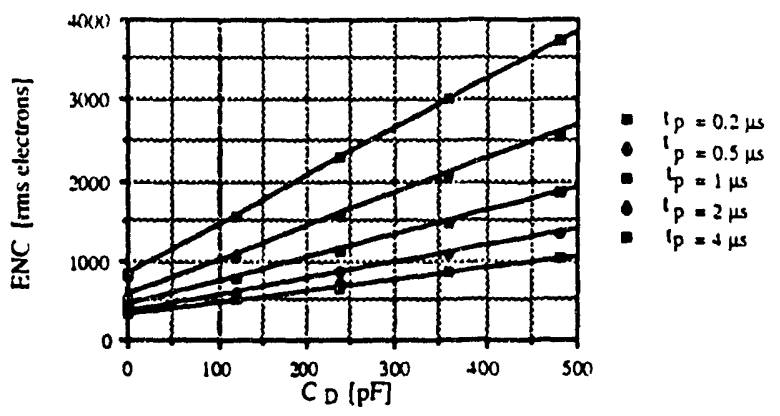

Fig. 5 - a) ENC as a function of the peaking time $t_{p}$, with the detector capacitance as a parameter.

b) ENC as a function of the detector capacitance $C_{D}$ at five different values of the peaking time $t_{p}$.

\section{V-RADIATION SENSITIVITY OF NOISE AND SMALL-SIGNAL PARAMETERS}

The radiation sensitivity of noise and small signal parameters has been tested by exposing the preamplifier to irradiation steps reaching a total integrated dose of $10 \mathrm{Mrad}$ of $\gamma$ - rays from a ${ }^{60}$ Co source.

After each irradiation step the input leakage current and the transconductance of the input JFET were monitored along with the preamplifier open-loop gain and the risetime relevant to the closed-loop, charge-sensitive configuration.

Meanwhile, the noise behaviour was tested by performing after each irradiation step the spectral analysis of the series noise referred to the input and by measuring the 
equivalent noise charge with the circuit connected as a charge-sensitive loop and followed by a semigaussian spectroscopy amplifier.

Irradiation has been carried out in both situations, of powered and unpowered preamplifier. Irradiation of the powered circuit has shown a sudden increase of the input leakage current, from $\approx 10 \mathrm{pA}$ to $\approx 1 \mathrm{nA}$ upon absorption of a step of comparatively small dose $(\approx 200 \mathrm{krad})$. The leakage current hasn't shown any further sizable increase throughout the irradiation programme, which reached a total dose of 10 Mrad. Such a behaviour in the leakage current is identical to that presented by discrete devices of the same geometry as $\mathrm{J} 1$.

The effect of irradiation on the series noise in the preamplifier is described in fig. 6 , which shows the voltage spectral density before and after irradiation.

In agreement with the results of previous radiation tests on JFET noise, the white noise is unaffected by the exposure to $\gamma$-rays, and this complies with the fact that no variations in transconductance were observed, while the low frequency portion of the spectrum increases in magnitude and changes in shape [4, 5].

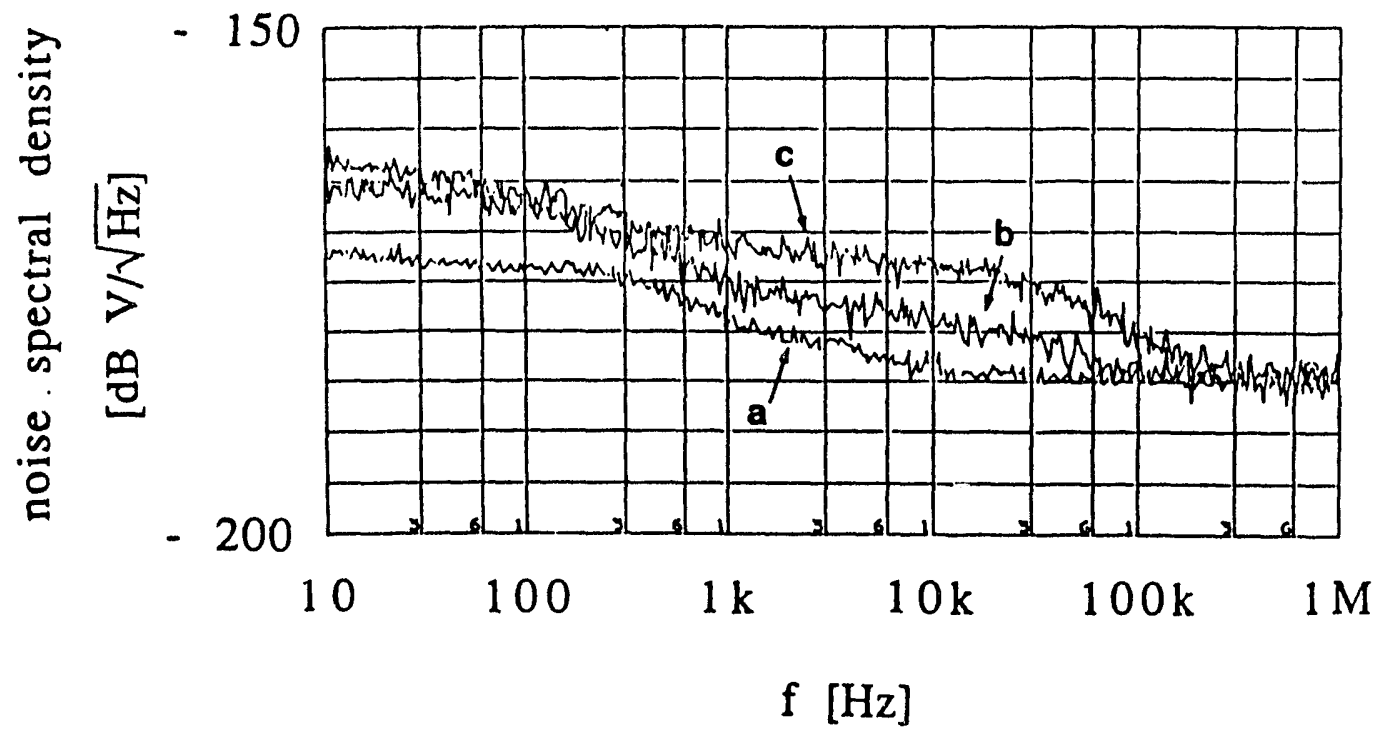

Fig. 6 - Spectral density of the series noise voltage as a function of frequency

a) before irradiation

b) after absorption of 2 Mrad integrated dose

c) after absorption of 10 Mrad integrated dose

The irradiation has been performed on the powered preamplifier $(0 \mathrm{~dB}$ corresponds to $1 \mathrm{~V} / \sqrt{\mathrm{Hz}}$ ). 
According to the previous discussion, it has to be expected that the irradiation is of very little effect on the equivalent noise charge measured at short processing times, where the white noise is of dominant importance. As the processing time is increased, ENC will become more and more affected by irradiation as a result of both effects: increased leakage current and enhanced noise density in the low frequency region. Such a behaviour is apparent in fig. 7, where ENC is plotted as a function of the peaking time $\tau_{P}$ of the semigaussian shaper employed to filter signal and noise at the preamplifier output.

Open-loop gain and the small signal risetime of the preamplifier connected in the charge-sensitive configuration are substantially unaffected by irradiation, as shown in Table II.

The behaviour of input leakage current, open-loop gain and risetime in the preamplifier irradiated in the power-off condition was found to be substantially identical to the previously discussed one. Also the variations in the series noise spectral densities observed in the preamplifier irradiated in the power-off situation do not deviate appreciably from those described in fig. 6.

It can be concluded that the behaviour of the monolithic preamplifier after exposure to $\gamma$-rays is close to that one could expect on the ground of the radiation sensitivity of discrete JFETs. The step, which is going to be raken now, is the exposure of the preamplifier to neutrons.

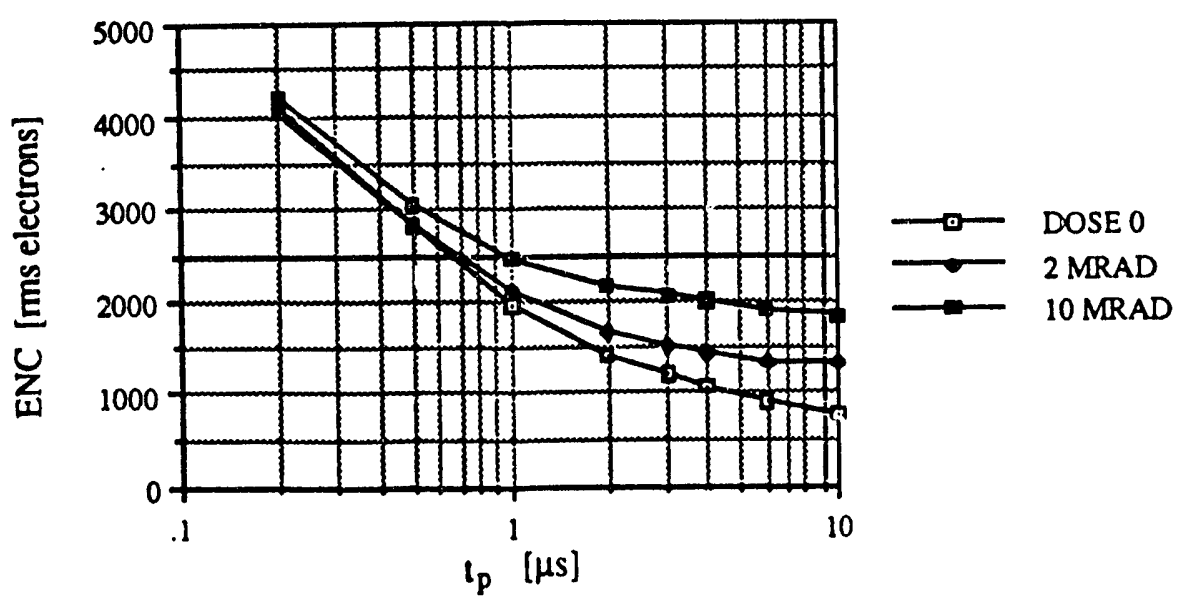

Fig. 7 - Equivalent noise charge as a funciion of the peaking time tp with the integrated dose as a parameter $\left(C_{D}=490 \mathrm{pF}\right)$. 


\section{VI-CONCLUSIONS}

A monolithic preamplifier has been realised on the base of a buried layer approach, by virtue of which epitaxial $\mathrm{N}$ - channel JFETs have been brought to co-exist on the same structure.

The results of measurements carried out on the preamplifier have shown that its noise and small-signal behaviour is very close to that expected from a discrete circuit employing the same type of JFETs. Upon absorption of $10 \mathrm{Mrad}$ integrated dose of $\gamma$ rays, the preamplifier has shown a negligible degradation in open-loop gain and risetime characteristics. A noise increase in the low-frequency region has been noticed, as an effect of inadiation. However, this does not sensibly affect the equivalent noise charge at peaking times shorter than about $0.5 \mu \mathrm{s}$.

\section{VII - ACKNOWLEDGEMENTS}

The authors acknowledge the contribution given by M. Hoye and L.A. Rehn of InterFET Co. throughout this work.

\section{REFERENCES}

1 - M. Demicheli, P.F. Manfredi, S. Rescia, V. Radeka, V. Speziali, Nucl. Instr. and Meth. in Phys. Res. A289 (1990) 418.

2 - P.F. Manfredi, V. Speziali, Radiat. Phys. Chem. Vol. 37, No. 4, p. 623, 1991.

3 - P.F. Manfredi, S. Rescia, V. Radeka, V. Speziali, IEEE Trans. Nucl Sci. NS38, 2, (1991), 83.

4 - J.H. Stephen, IEEE Trans. Nucl. Sci. NS-33, 6, (1986), 1465.

5 - V. Radeka, S. Rescia, L.A. Rehn, P.F. Manfredi, V. Speziali: Monolithic Junction Field-Effect Transistor Charge-Sensitive Preamplifier for Calorimetry at High Luminosity Hadron Colliders. Paper presented at IEEE 1991 Nuclear Science Symposium, Santa Fe, New Mexico, November 2-9, 1991. 
Table I

Preamplifier Characteristics

(substrate voltage $+20 \mathrm{~V}$ )

Open loop dc gain

Input device gate width W

Open loop input capacitance

Input current $\left(\mathrm{T}=300^{\circ} \mathrm{K}\right)$

Supply voltages

Full scale output

linear range

Power consumption

Die size
$>85 \mathrm{~dB}$

$11.000 \mu \mathrm{m}$

$65 \mathrm{pF}$

$\sim 10 \mathrm{pA}$

$+12 \mathrm{~V}-6 \mathrm{~V}$

$2 \mathrm{~V}$ on a $1 \mathrm{k} \Omega$ load

either output polarity

$230 \mathrm{~mW}$

$2.5 \mathrm{~mm} \times 2.5 \mathrm{~mm}$. 
Table II

Radiation sensitivity of small-signal parameters

Absorbed dose, ${ }^{60} \mathrm{Co} \gamma$-rays

Open-loop gain

Small signal risetime
0

$10 \mathrm{Mrad}$

$87.4 \mathrm{~dB}$

$87.4 \mathrm{~dB}$

$37 \mathrm{~ns}$

$39 \mathrm{~ns}$ 


\section{FIGURE CAPTIONS}

Fig. 1 - a) Monolithic preamplifier structure.

b) Charge-sensitive configuration showing the externally added components.

Fiz. 2 - a) Open-loop gain and phase characteristics as functions of frequency.

b) Open-loop gain characteristics at two different values of the substrate voltage (continuous line: $\mathrm{V}_{\text {substratc }}=+12 \mathrm{~V}$; dotted line: $\left.\mathrm{V}_{\text {substrate }}=+20 \mathrm{~V}\right)$.

Fig. 3 - Preamplifier response to a delta-impulse input current for a $500 \mathrm{pF}$ detector capacitance and a $10 \mathrm{pF}$ feedback capacitance (for $\mathrm{C}_{\mathrm{F}}=33 \mathrm{pF}$ used in the tests of fast liquid argon calorimeters, the time scale is reduced by a factor of 3.3).

Fig. 4 - Spectral density of the noise voltage referred to the preamplifier input as a function of frequency $(0 \mathrm{~dB}$ corresponds to $1 \mathrm{~V} / \sqrt{\mathrm{Hz}})$.

At $\mathrm{f}>10^{4} \mathrm{~Hz} \mathrm{e}_{\mathrm{n}} \equiv 0.63 \mathrm{nV} / \sqrt{\mathrm{Hz}}$.

Fig. 5 - a) ENC as a function of the peaking time $t_{p}$, with the detector capacitance as a parameter.

b) ENC as a function of the detector capacitance $C_{D}$ at five different values of the peaking time $t_{p}$.

Fig. 6 - Spectral density of the series noise voltage as a function of frequency

a) before irradiation

b) after absorption of 2 Mrad integrated dose

c) after absorption of $10 \mathrm{Mrad}$ integrated dose

The irradiation has been performed on the powered preamplifier $(0 \mathrm{~dB}$ corresponds to $1 \mathrm{~V} / \sqrt{\mathrm{Hz}}$ ). 
Fig. 7 - Equivalent noise charge as a function of the peaking time $t_{p}$ with the integrated dose as a parameter $\left(C_{D}=490 \mathrm{pF}\right)$. 

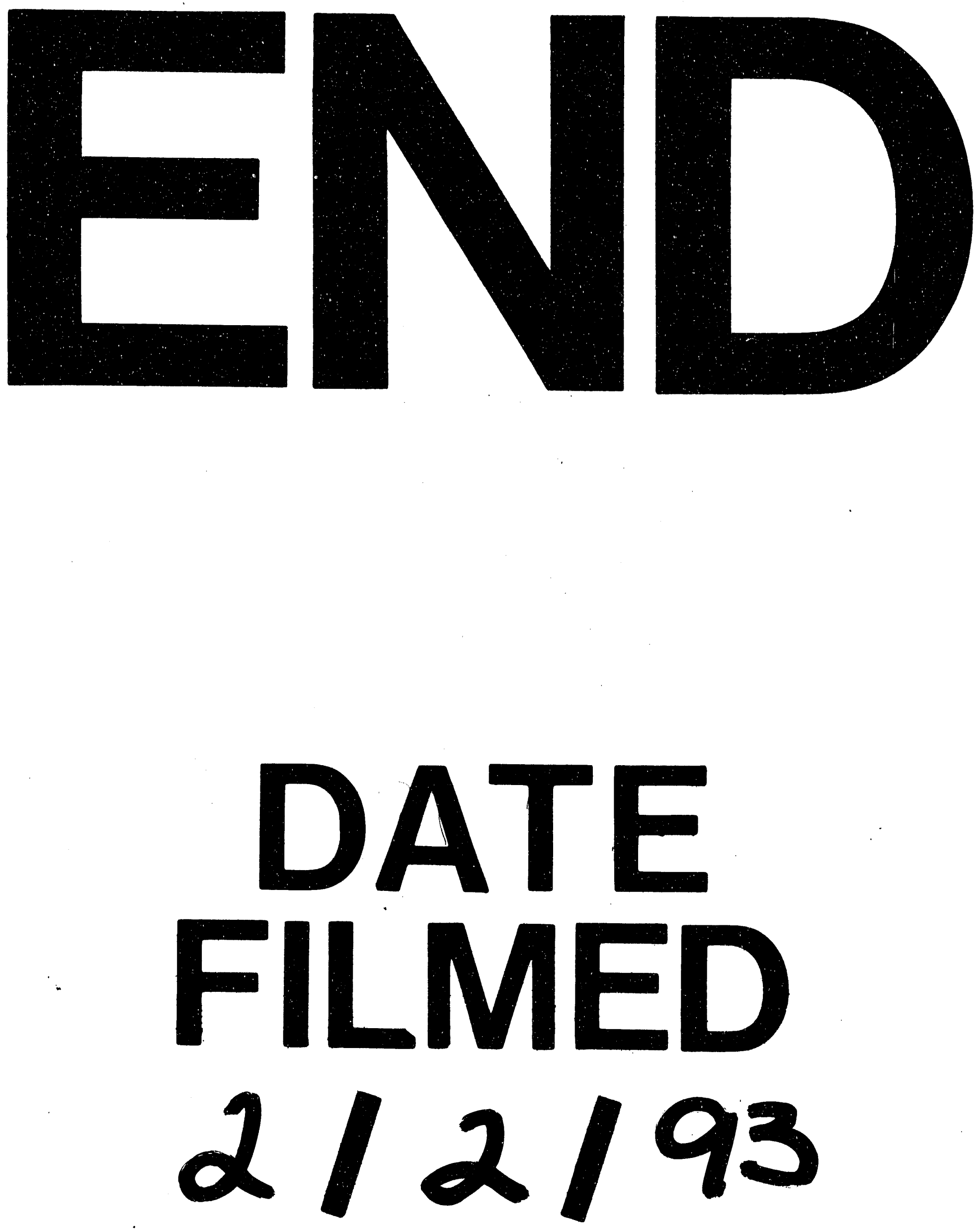
